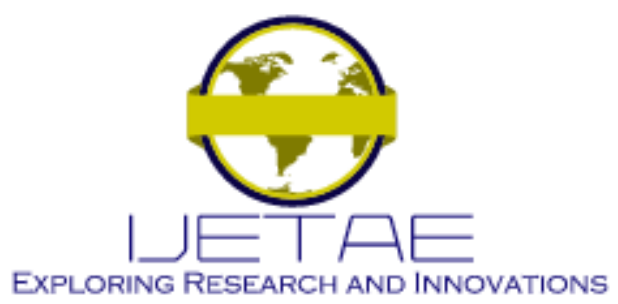

International Journal of Emerging Technology and Advanced Engineering

Website: www.ijetae.com (E-ISSN 2250-2459, Scopus Indexed, ISO 9001:2008 Certified Journal, Volume 11, Issue 11, November 2021)

Manuscript Received: 08 October 2021, Received in Revised form: 05 November 2021, Accepted: 12 November 2021

DOI: $10.46338 /$ ijetae1121_22

\title{
Improved Imaging Model in the Presence of Multiplicative Spatially Extended Cloaking Interference
}

\author{
Hennadii Khudov ${ }^{1}$, Igor Ruban ${ }^{2}$, Oleksandr Makoveichuk ${ }^{3}$, Yevhen Stepanenko ${ }^{4}$, Irina Khizhnyak ${ }^{5}$, \\ Sergey Glukhov ${ }^{6}$, Olesya Symkanych ${ }^{7}$, Yuriy Solomonenko ${ }^{8}$, Andrii Baranov ${ }^{9}$, Nazar Shamrai ${ }^{10}$, \\ Rostyslav Khudov ${ }^{11}$ \\ ${ }^{1,5,8,10}$ Ivan Kozhedub Kharkiv National Air Force University, Ukraine, Kharkiv, 61023 \\ ${ }^{2,3}$ Kharkiv National University of Radio Electronics, Ukraine, Kharkiv, 61166 \\ ${ }^{4,6}$ Military Institute of Taras Shevchenko Kyiv National University, Ukraine, Kyiv, 03189 \\ ${ }^{7}$ Uzhhorod National University, Ukraine, Uzhhorod, 88000 \\ ${ }^{9}$ Hetman Petro Sahaidachnyi National Army Academy, Ukraine, Lviv, 79026 \\ ${ }^{11}$ V. N. Karazin Kharkiv National University, Ukraine, Kharkiv, 61022
}

\begin{abstract}
The paper proposes an improved imaging model in the presence of multiplicative spatially extended cloaking interference. The model take into account the effect of multiplicative masking interference. To simplify the calculations of the image brightness in the distorted region the diagram technique is used. Unlike the known ones, the model takes into account the concentration of the distorting medium in a narrow squat layer, the primary reflection of solar radiation from the upper boundary of the distorting layer and subsequent multiple re-reflections of the transmitted radiation of the visible wavelength range from the earth's surface and the upper boundary of the distorting medium layer. A technique for finding and taking into account the reflection and re-reflection coefficients of radiation to restore distorted images is proposed. The results of experimental studies are presented. For the experiment, the image of the territory of Iraq during the 2003 "Freedom for Iraq" hostilities was selected.
\end{abstract}

Keywords - image, model, multiplicative, extended cloaking interference, spacecraft, reflection, coefficient

\section{INTRODUCTION}

At present, high requirements are imposed on the quality of image processing obtained from onboard optoelectronic observation systems to ensure their processing [1]. First of all processing under conditions of natural and artificial masking interference. Known methods of processing under the influence of masking interference are effective only for certain spectral ranges. The use of well-known image processing methods does not always give an acceptable result [1-2].
This, in turn, has a negative impact on the subsequent stages of image processing (for example, segmentation and object detection in images) [3-4].

Primarily the adopted imaging model determines the quality of the image processing methods. Existing imaging models take into account the effects of additive noise and interference. In this case, additive noise and noise affect specific pixels. The spatial extent of the interference is not taken into account. The effects of multiplicative interference are not accounted for known imaging models.

Also, there is no allowance for the spatial extent of the multiplicative interference. Thus, the task of developing an improved model of image formation under conditions of exposure to extended masking interference is urgent.

\section{LiteRATURE REVIEW AND PROBLEM STATEMENT}

When forming images of observation from the spacecraft, the atmosphere is a disturbing medium. The atmosphere distorts satellite data. In some parts of the electromagnetic range, for example, in the far infrared with a wavelength of about $100 \mu \mathrm{m}$, the atmosphere generally prevents the receipt of information [1].

Richards J. [2] notes that various aerosols significantly affect the formation of images. Natural aerosols, solid and liquid particles suspended in the air include space, volcanic and soil mineral dust, plant pollen, sea salt particles, cloud and fog droplets, and smoke particles from forest and peat fires. Anthropogenic aerosol is particles of soot, ash, cement and other production and transport waste. 


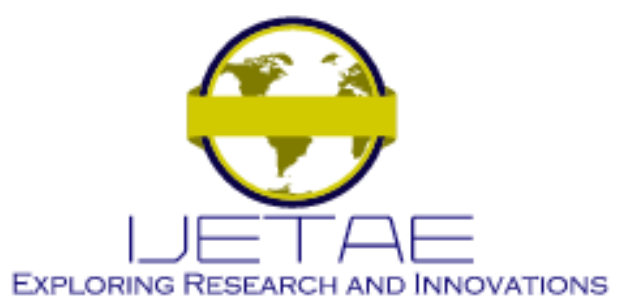

International Journal of Emerging Technology and Advanced Engineering

Website: www.ijetae.com (E-ISSN 2250-2459, Scopus Indexed, ISO 9001:2008 Certified Journal, Volume 11, Issue 11, November 2021)

Significant sources of pollution are furnaces, hydroelectric power plants, heat engines. However, the results [2] are not taken into account in imaging models.

Karamti H. et. Al. [5] note that the main types of interference are impulse noise (Fig. 1) and "stripe" (Fig. 2). Impulse noise is large single isolated outliers (isolated contrast points) (Fig. 1). The stripes appear as quasiperiodic changes in the pixel intensity along only one coordinate axis (Fig. 2). In [5] image models are developed that take into account impulse noise and "stripe" noise. Formation of the image model in terms of multiplicative noise is not considered.

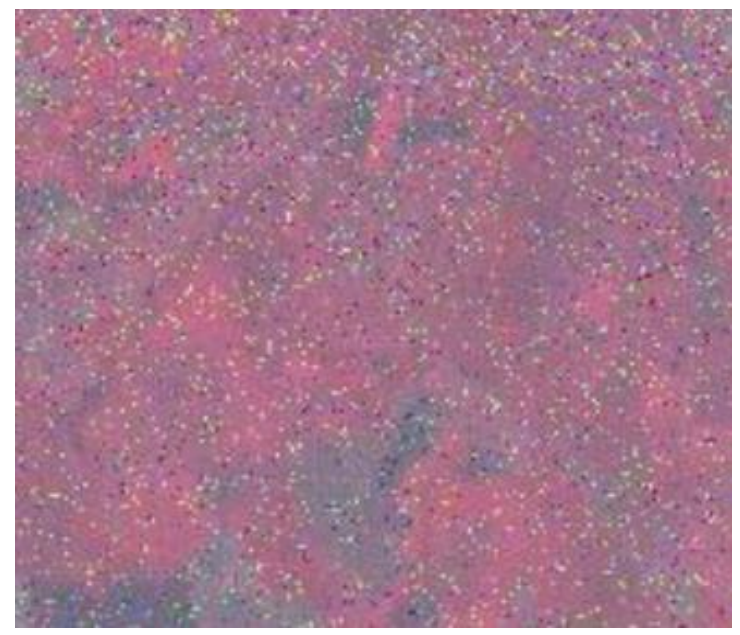

Fig. 1 Image with impulse noise interference

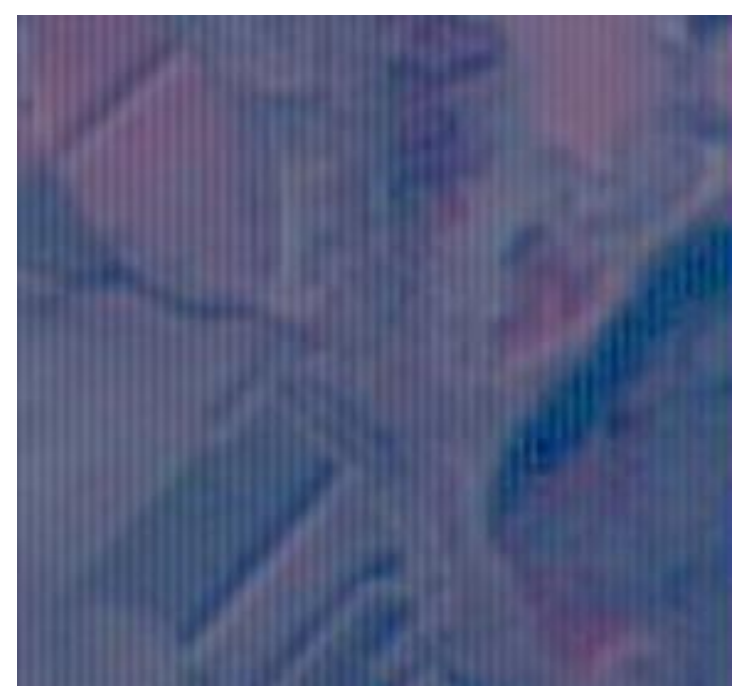

Fig. 1 Image with "stripe"interference
Ballish, Bradley A. et. Al. [6] elaborate that the imaging model takes into account that the atmosphere attenuates upward radiation from the Earth's surface and in the transparency bands. This effect is described by the volume absorption coefficient $\beta$. Light is scattered by gas molecules, water droplets, dust particles, this effect is described by the volume scattering coefficient $\sigma$.

Ruban I. et. Al. [7] consider that optical thickness $\tau$ is the product of the volumetric coefficient of light attenuation by the atmosphere by the geometric path length of the light beam. If the coefficients $\beta$ and $\sigma$ depend on the coordinate (and the density of the atmosphere changes with height), then on the path between the points with coordinates $r_{1}$ and $r_{2}$, the optical thickness $\tau$ is (1) [7]:

$$
\tau\left(r_{1}, r_{2}\right)=\int_{r_{1}}^{r_{2}}(\beta(r)+\sigma(r)) d r
$$

When the effects of multiple scattering are small, i.e. within the limits of validity of Bouguer's law, we have (2) [7]:

$$
I_{1}=I_{0} e^{-\tau}
$$

where $\mathrm{I}_{0}$ is the radiation intensity at the initial point (on the Earth's surface);

$I_{1}$ is the radiation intensity at the final point (on the satellite orbit);

$\tau$ is a optical thickness (the natural logarithm of the ratio of incident to transmitted radiant power through a material [8-9]).

Bouguer's law (formula (2)) shows that absorption increases extremely rapidly with increasing layer thickness $(\tau)$.

Multiple scattering effects can be neglected for the green $(\mathrm{G})$ and longer wavelength regions of the spectrum, but not for the blue (B) region. The formula (2) is valid for the observation in nadir. If the observation is carried out at an angle $\delta$ to the nadir, then for a homogeneous atmosphere the radiation intensity at the final (in the satellite orbit) point is calculated by the formula (3) [7]:

$$
I_{2}=I_{0} e^{-\left(\frac{\tau}{\sin \delta}\right)} .
$$




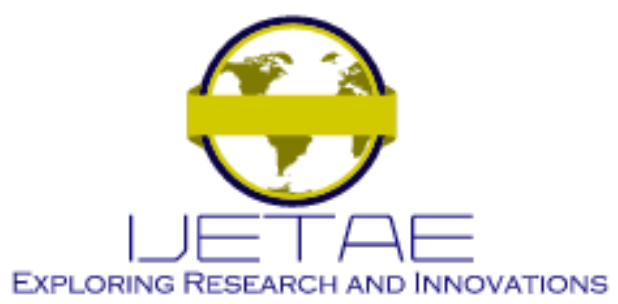

International Journal of Emerging Technology and Advanced Engineering

Website: www.ijetae.com (E-ISSN 2250-2459, Scopus Indexed, ISO 9001:2008 Certified Journal, Volume 11, Issue 11, November 2021)

The formula (3) does not take into account the curvature of the Earth, however, there are methods [10] that allow such an account. The influence of possible horizontal local inhomogeneities (the presence of fog, haze, dust clouds, etc.) is also not taken into account. Therefore, calculation (3) is approximate.

Laven $\mathrm{Ph}$. [9] notes that the scattering of light by gas molecules, water droplets, dust grains obeys certain laws. The intensity of molecular scattering for light with a wavelength $\lambda$ is proportional to $\lambda^{-4}$, the effect is most noticeable in the short-wavelength part of the spectrum, it is responsible for the blue color of the sky. Scattering by aerosols (particle size from $0.1 \lambda$ to $10 \lambda$ ) leads to a weaker dependence of the intensity on the wavelength $\lambda^{-n}$, where $(0<\mathrm{n}<4)$. Particles of smoke and clouds have sizes much larger than the wavelengths of the visible and infrared ranges, there is no dependence of the intensity of scattered solar radiation on the wavelength, the color of smoke and clouds is close to white.

The spacecraft can receive information from large areas. In this case, different parts of the surface are observed at different angles $\delta$, and the beam from them travels different distances in the atmosphere. As a result, areas of the surface with the same optical properties can have different brightness. For calculations using the Bouguer's formula, it is necessary to know the optical thickness of the atmosphere $\tau$ [12-13]. This can be done by measuring the attenuation of solar radiation on Earth. From the satellite you can estimate the brightness of objects with a priori known optical characteristics. It is also possible to roughly estimate $\tau$ in the visible part of the spectrum using the relationship between $\tau$ and the meteorological characteristics of the troposphere - air temperature, wind speed, relative humidity and water vapor pressure [12-13]. Bradley M. and Gasseler M. [12] note that the influence of the atmosphere can be taken into account more accurately on the basis of the theory of radiation transfer in the atmosphere. This theory makes it possible to estimate the brightness of objects from the measurement data of the upward radiation by solving the inverse problem.

Christensen M. et. Al. [14] consider that aerosols enhance cloud lifetime and brightness along the stratusto-cumulus transition. They consider all cloud droplets on Earth form from tiny airborne particles known as aerosols.
Using a combination of time-lapse satellite imagery and air mass trajectory modeling, Christensen M. et. Al. [14] show that aerosols can enhance cloud fraction and extend the lifetime of overcast cloud fields.

Authors [15] determine the flight routes of unmanned aerial vehicles (UAV). The determining of the flight routes of UAV base on swarm methods. Formation of the image model in terms of multiplicative noise is not considered.

Authors [16-17] discuss medical image processing. Authors [16-17] present the results of cytological image processing by k-means, Prewitt, Sobel, Canny, Hough methods and combinations of these methods. A comparative visual and quantitative assessment of the quality of cytological image processing was performed. However, authors [16-17] don't take into account the features of image formation from onboard observation systems.

Authors [18] present the model and the method for forming a mosaic sustainable marker of augmented reality. Authors [19] present the mosaic sustainable marker detection method for augmented reality systems. Authors [20] present the mosaic sustainable marker model for augmented reality systems. Results [18-20] are only applicable in augmented reality systems. Papers [18-20] don't take into account the peculiarities of image formation from onboard surveillance systems.

Authors [21-23] consider the processing of images from onboard optical-electronic surveillance systems. In this case, the authors [21-23] don't take into account the presence of noise in the images.

Thus, the known image formation model can be represented as (4) [7, 12, 13]:

$$
v(x, y)=I(x, y) f(x, y),
$$

where for each point with coordinates $(x, y)$ the functions are introduced:

$\mathrm{v}(\mathrm{x}, \mathrm{y})$ is the illumination at a point with coordinates $(\mathrm{x}, \mathrm{y})$;

$\mathrm{I}(\mathrm{x}, \mathrm{y})$ is the external illumination on the path of propagation of radiation from the Sun to a point with coordinates $(\mathrm{x}, \mathrm{y})$, a change in which (attenuation) creates the effect of an extended masking interference;

$f(x, y)$ is the coefficient of reflection from the underlying surface with the objects of observation located on it. 


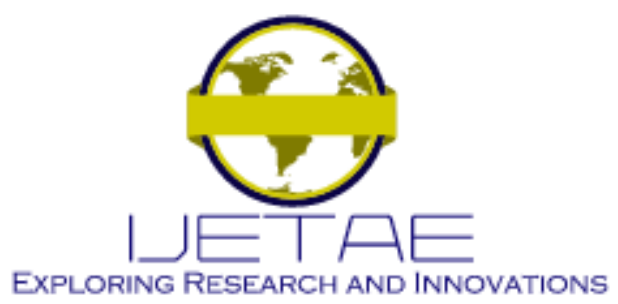

International Journal of Emerging Technology and Advanced Engineering

Website: www.ijetae.com (E-ISSN 2250-2459, Scopus Indexed, ISO 9001:2008 Certified Journal, Volume 11, Issue 11, November 2021)

The image registration model can be written as (5) [7]:

$$
\mathrm{g}(x, y)=s(x, y) v(x, y)+n(x, y)
$$

where $\mathrm{g}(\mathrm{x}, \mathrm{y})$ is the recorded illumination of a point with coordinates;

$\mathrm{s}(\mathrm{x}, \mathrm{y})$ is the coefficient of change in the illumination of the image on the track "point with coordinates $(\mathrm{x}, \mathrm{y})-$ spacecraft" (the effect of extended interference);

$\mathrm{n}(\mathrm{x}, \mathrm{y})$ is the noise.

Thus, taking into account (4) and (5), with respect to the function $f(x, y)$, the functions $I(x, y)$ and $s(x, y)$ are multiplicative. However, the known imaging models do not take into account the form of functions $\mathrm{I}(\mathrm{x}, \mathrm{y})$ and $\mathrm{s}(\mathrm{x}, \mathrm{y})$. The multiplicativity of functions $\mathrm{I}(\mathrm{x}, \mathrm{y})$ and $\mathrm{s}(\mathrm{x}, \mathrm{y})$ is also not taken into account.

\section{MATERIALS AND RESEARCH METHODS}

Let us take into account that for the visible range of waves, the attenuation of optical radiation by the atmosphere occurs due to the scattering of radiation energy due to an optically inhomogeneous atmosphere. Let us assume that the effect of the multiplicative interference during observation from the spacecraft is concentrated in a narrow surface layer of the atmosphere. We consider a narrow surface layer of the atmosphere to be homogeneous. The distorting factor will be simulated by multiple reflections from an effective semitransparent screen, which is located on the layer separation line. The coefficient of reflection from the screen is assumed to be set and equal to $\alpha$, the attenuation of light in the screen itself, between the screen and the underlying surface, is taken into account by the factor $\gamma$.

In the absence of interference, the recorded image $g(x, y)$ is mainly determined by the reflection from the Earth's surface. Let us assume for simplicity that the illumination $\mathrm{T}$ is constant, then it follows from (4) that in the undistorted region (formula (6)):

$$
\mathrm{g}(x, y)=\text { const } \cdot f(x, y),
$$

where $f(x, y)$ is the brightness of the original image with the objects of observation located on it.

Without loss of generality, we can further assume that in (6), in this case, the values of all quantities used below will lie in the range $[0 \ldots 1]$.
To simplify the calculations of the image brightness in the distorted region, it is convenient to use the diagram technique. Thus, multiple reflections can be represented as the following row (the top line corresponds to the screen) (Fig. 3).

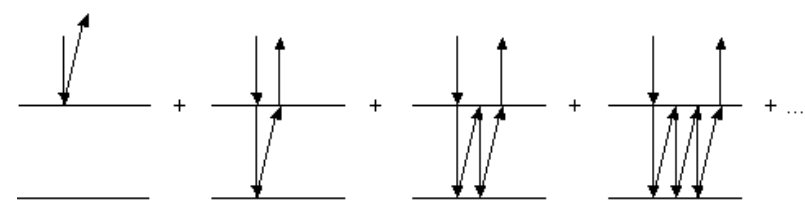

Fig. 3 The diagram technique for calculating image brightness

The resulting brightness of the recorded image at each point $(\mathrm{x}, \mathrm{y})$ in the region of the presence of distortions is found as the sum of terms, each of which corresponds to a single reflection, double, triple, etc. In this case, each reflection from the screen introduces a multiplier $\alpha$, passage through the screen - a factor (1- $\alpha)$, reflection from the underlying surface - $\mathrm{f}(\mathrm{x}, \mathrm{y})$, each passage between the screen and the underlying surface gives a factor $\gamma$.

Thus, diagram (Fig. 3) will correspond to the series (7):

$$
\begin{aligned}
& g(x, y)=\alpha+(1-\alpha) \gamma f(x, y) \gamma(1-\alpha)+ \\
& +(1-\alpha) \gamma f(x, y) \gamma \alpha \gamma f(x, y) \gamma(1-\alpha)+ \\
& +(1-\alpha) \gamma f(x, y) \alpha \gamma f(x, y) \gamma \alpha \gamma f(x, y) \gamma(1-\alpha)+\mathrm{K}
\end{aligned}
$$

where $\gamma$ is the transmission coefficient in the area between the screen and the underlying surface (in the absence of interference $\gamma=1$ ).

Series (7), starting from the second term (the first term is equal $\alpha$ ), is a geometric progression with the first term $(1-\alpha)^{2} \gamma^{2} f(x, y)$ and denominator $\alpha \gamma^{2} f(x, y)<1$ The sum of the series is (8) [24]:

$$
g(x, y)=\alpha+\frac{(1-\alpha)^{2} \gamma^{2} f(x, y)}{1-\alpha \gamma^{2} f(x, y)} .
$$

Thus, to solve the problem of image restoration from the measured values of $\mathrm{g}(\mathrm{x}, \mathrm{y})$, it is necessary to estimate the parameters of the model $\alpha, \gamma$, after which the $\mathrm{f}(\mathrm{x}, \mathrm{y})$ is determined from (8). The calculated value of the $f(x, y)$ image (reconstructed image) will hereinafter be referred to as $\mathrm{r}(\mathrm{x}, \mathrm{y})$.

Solving equation (8) with respect to the $f(x, y)$ we find that in the distorted region (formula (9)): 


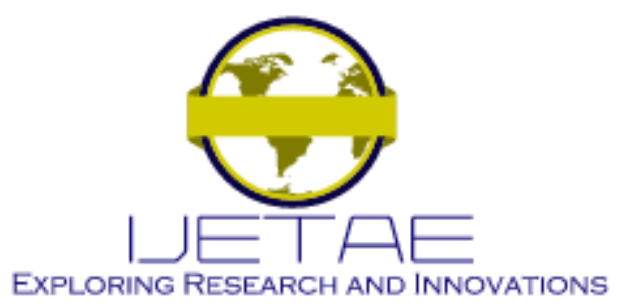

International Journal of Emerging Technology and Advanced Engineering

Website: www.ijetae.com (E-ISSN 2250-2459, Scopus Indexed, ISO 9001:2008 Certified Journal, Volume 11, Issue 11, November 2021)

$$
r(x, y)=\frac{1}{\gamma^{2}} \frac{g(x, y)-\alpha}{1+\alpha g(x, y)-2 \alpha} .
$$

Thus, we finally get (10):

$$
r(x, y)=\left\{\begin{array}{l}
\frac{1}{\gamma^{2}} \frac{g(x, y)-\alpha}{1+\alpha g(x, y)-2 \alpha}, \text { for distortion area } ; \\
g(x, y), \text { for undistorted area } .
\end{array}\right.
$$

The estimation of the reflection coefficient from the distorting layer $\alpha(\mathrm{x}, \mathrm{y})$ for each point of the recorded image of the $\mathrm{g}(\mathrm{x}, \mathrm{y})$ is carried out in a sliding rectangular window $\mathrm{g}_{\mathrm{w}}(\mathrm{x}, \mathrm{y})$. In this case it is taken into account that the coefficient of reflection from the distorting layer of the explosive $\alpha$ and the coefficient of transmission of the explosive $\gamma$ for each point of the recorded image are functionally and statistically independent quantities. Therefore they are evaluated separately. By varying the parameter $\alpha$ in the range of $[0 \ldots 1]$ and $\gamma=$ const, we obtain a number of reconstructed images $r_{w}=r_{w}(\alpha)$.

It is assumed that the estimate of the parameter $\alpha$ is its value $\alpha^{\prime}$ at which the variance $D$ of the image fragment reconstructed according to formula (9) in the window, $\mathrm{r}_{\mathrm{w}}=\mathrm{r}_{\mathrm{w}}(\alpha)$ takes the maximum value (11):

$$
\alpha^{\prime}=\arg \max D\left(r_{W}(\alpha)\right)
$$

In (11) the variance $\mathrm{D}$ is considered as a function $\mathrm{D}\left(\mathrm{r}_{\mathrm{w}}(\alpha)\right)$ of the argument $\alpha$.

The coefficient $\gamma(\mathrm{x}, \mathrm{y})$ is chosen so that the average value of the brightness in the reconstructed area of the image is equal to the average in the undistorted area.

Let us illustrate the above with the following example. Fig. 4 shows the image of the territory of Iraq during the 2003 "Freedom for Iraq" hostilities, taken from the website of the Space Imaging Company [25]. The image (Fig. 4) was obtained using the Ikonos optical-electronic surveillance system. Fig. 4 shows the multiplicative interference. The multiplicative interference is caused by the haze from an oil tank fire.
Unlike additive, multiplicative interference is a nonlinear effect and causes non-linear signal distortion. Underestimating the level of multiplicative noise causes large gaps in the contours of image objects when solving the problem of contour segmentation. This, in turn, affects the recognition error of these objects.

Three characteristic rectangular fragments were selected in the undistorted (fragments 1-3) and distorted (fragments 4-6) areas of the image. The size of each slice is equal to $(128 \times 128)$ pixels.

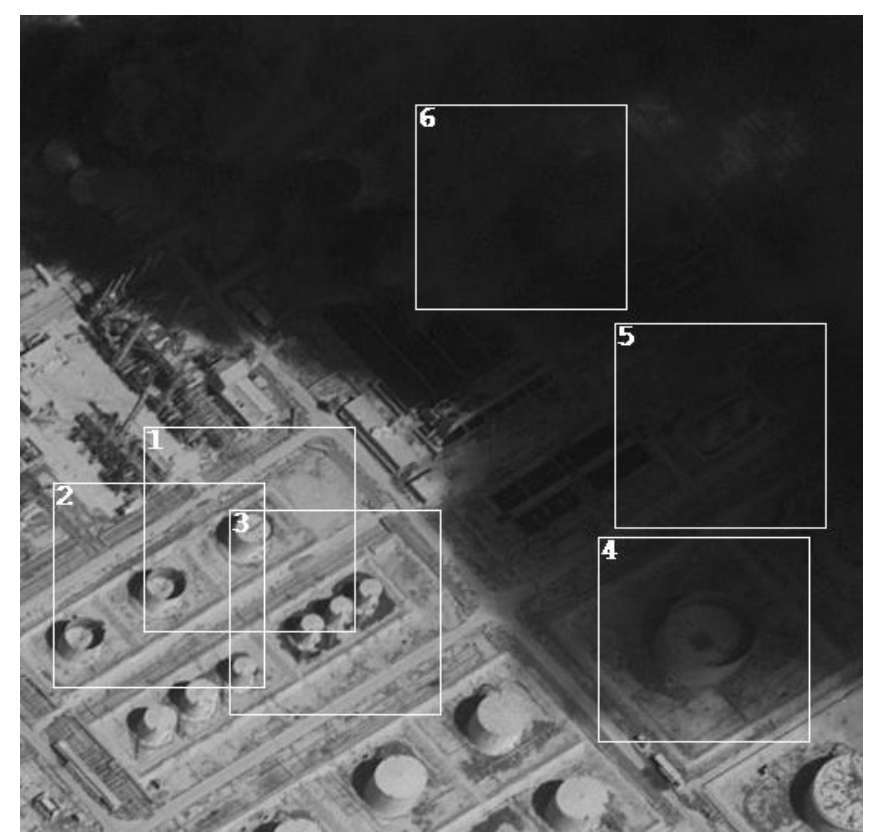

Fig. 4 The image of the territory of Iraq during the 2003 "Freedom for Iraq" hostilities with the undistorted (fragments 1, 2,3) and distorted (fragments $4,5,6$ ) areas of the image

Fig. 5-10 show fragments of the image (Fig. 4) and plotted the graphs of the dependence of the variance of the reconstructed image on the parameter value $\alpha$ for the image area undistorted by noise (fragments 1-3, Fig. 5-7) and for the image area distorted by noise (fragments 4-6, Fig. 8-10). 


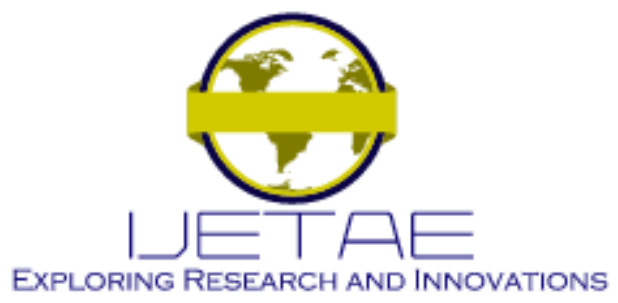

International Journal of Emerging Technology and Advanced Engineering

Website: www.ijetae.com (E-ISSN 2250-2459, Scopus Indexed, ISO 9001:2008 Certified Journal, Volume 11, Issue 11, November 2021)

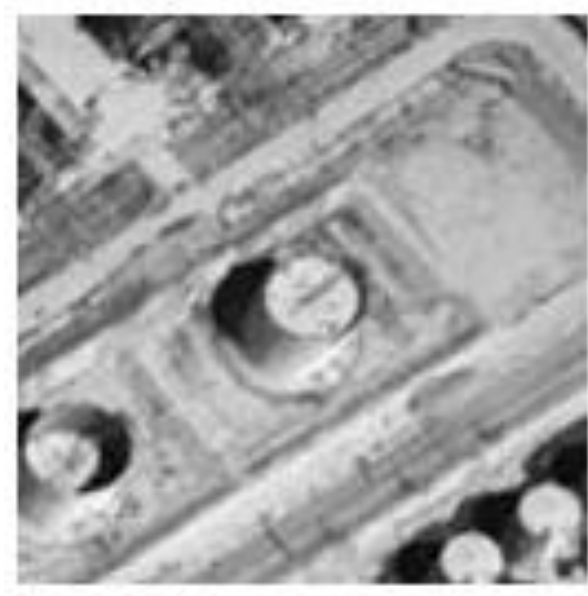

a)

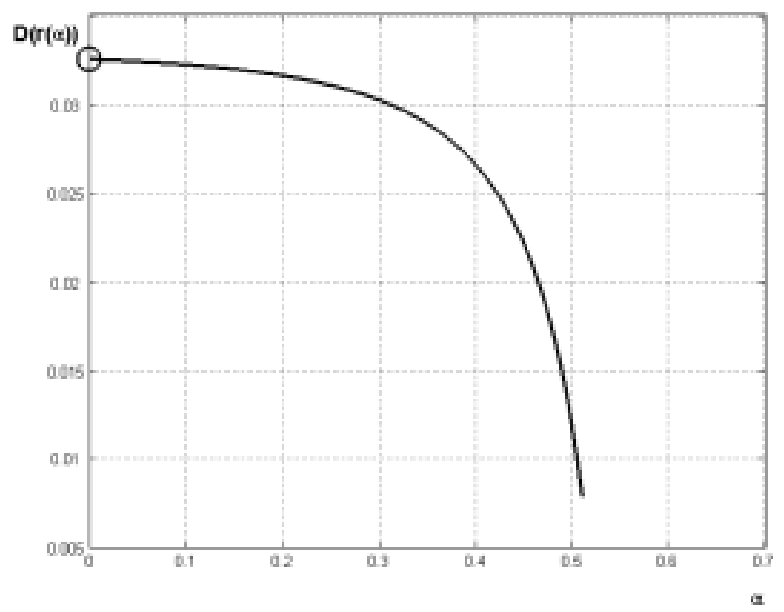

b)

Fig. 5 Fragment 1: a) image of fragment 1; b) graph of the dependence of the variance $D(r(\alpha))$ of the reconstructed image on the parameter value $\alpha$

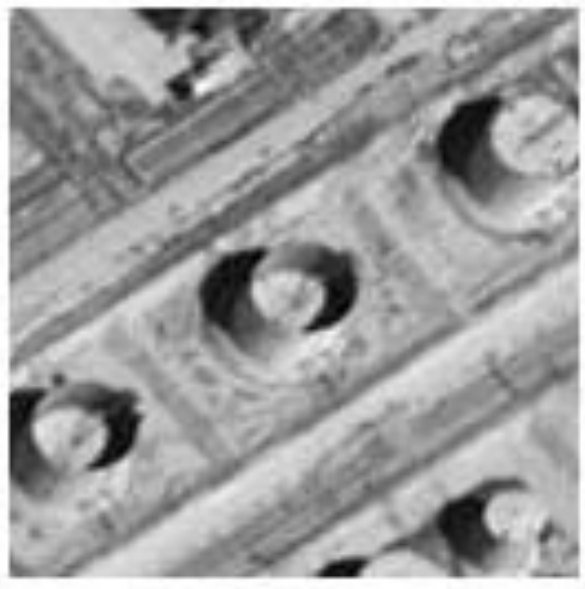

a)

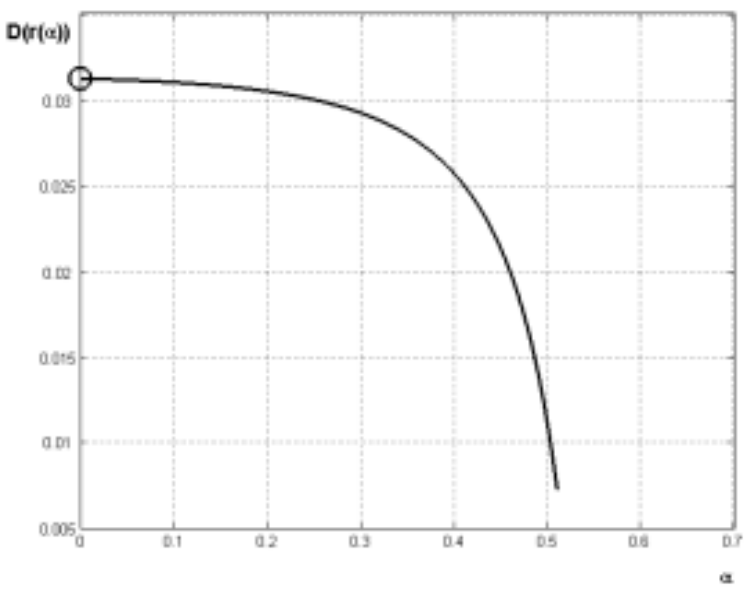

b)

Fig. 6 Fragment 2: a) image of fragment 2; b) graph of the dependence of the variance $D(r(\alpha))$ of the reconstructed image on the parameter value $\alpha$ 


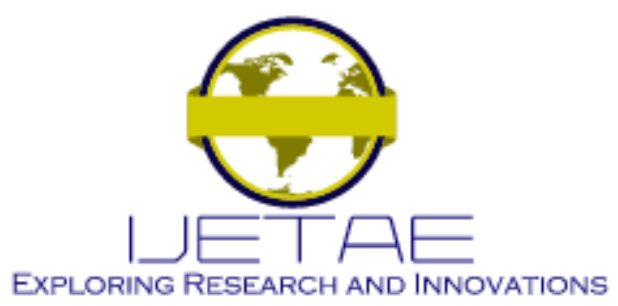

International Journal of Emerging Technology and Advanced Engineering

Website: www.ijetae.com (E-ISSN 2250-2459, Scopus Indexed, ISO 9001:2008 Certified Journal, Volume 11, Issue 11, November 2021)

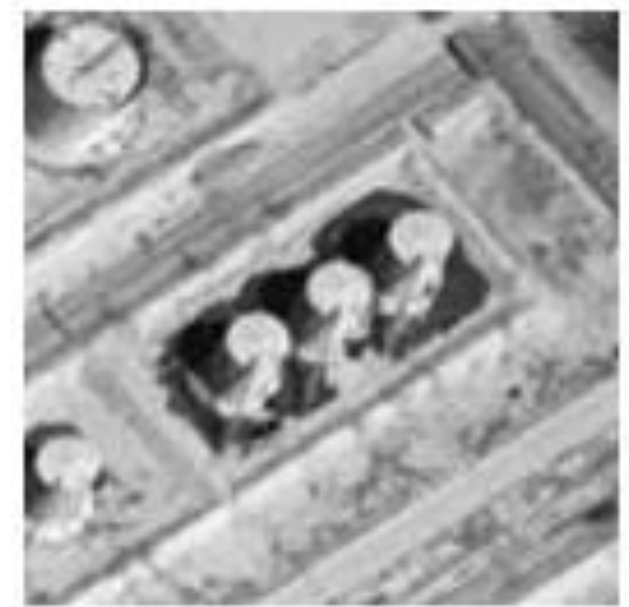

a)

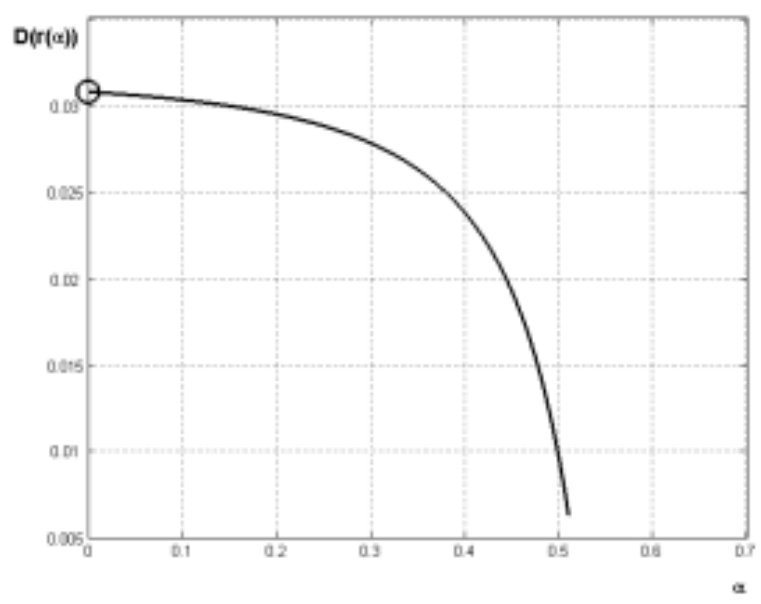

b)

Fig. 7 Fragment 3: a) image of fragment 3; b) graph of the dependence of the variance $D(r(\alpha))$ of the reconstructed image on the parameter value $\alpha$

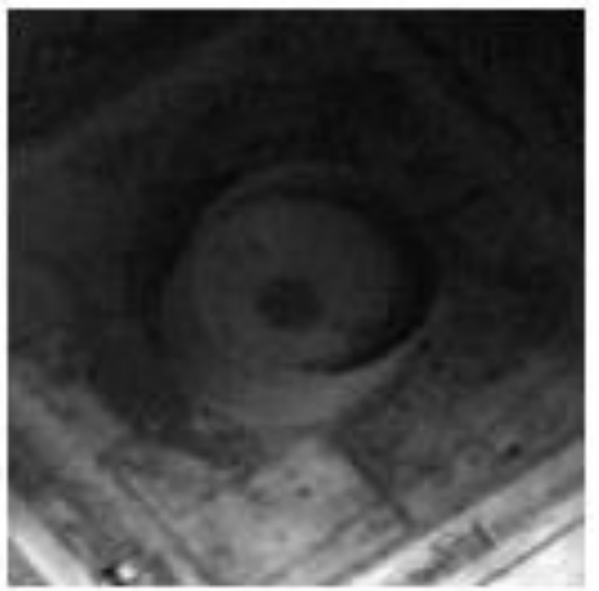

a)

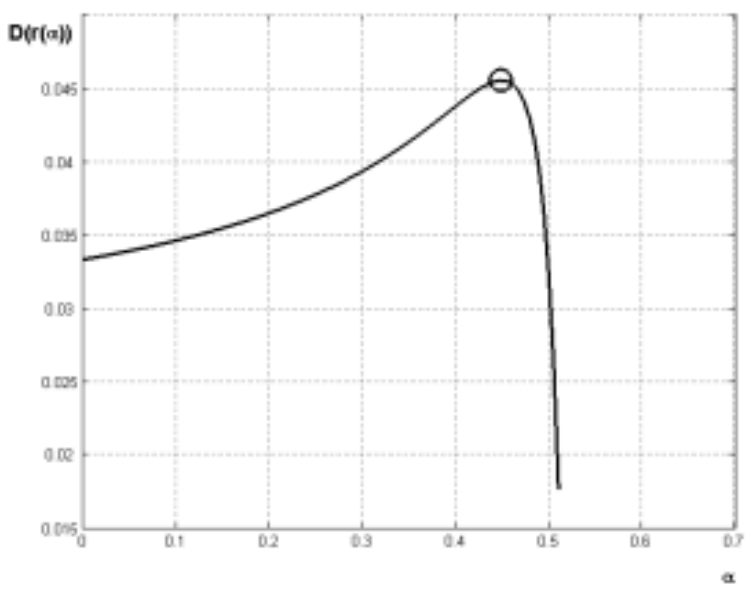

b)

Fig. 8 Fragment 4: a) image of fragment 4; b) graph of the dependence of the variance $D(r(\alpha))$ of the reconstructed image on the parameter value $\alpha$ 


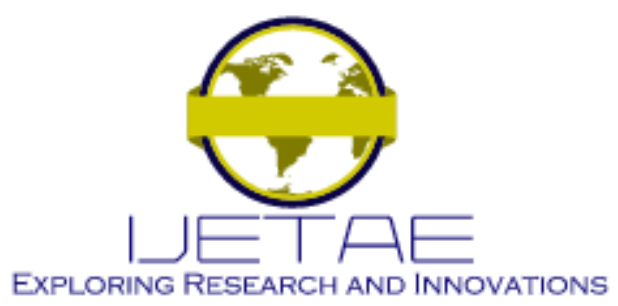

International Journal of Emerging Technology and Advanced Engineering

Website: www.ijetae.com (E-ISSN 2250-2459, Scopus Indexed, ISO 9001:2008 Certified Journal, Volume 11, Issue 11, November 2021)

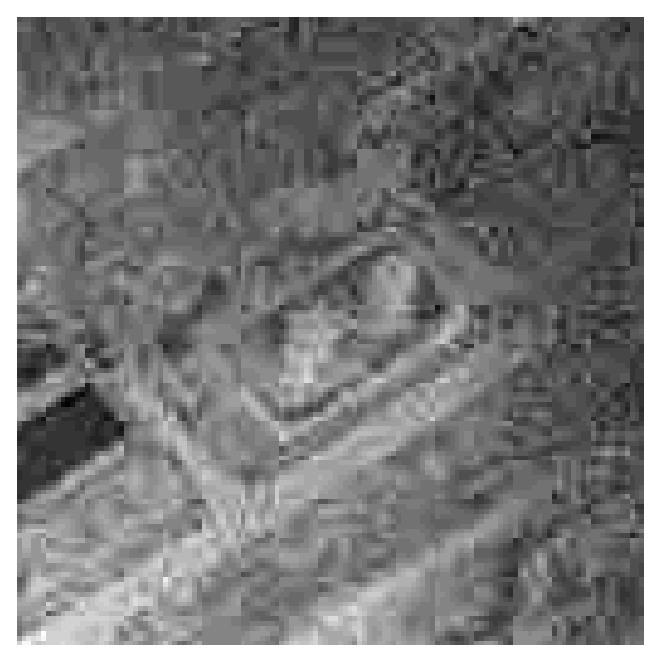

a)

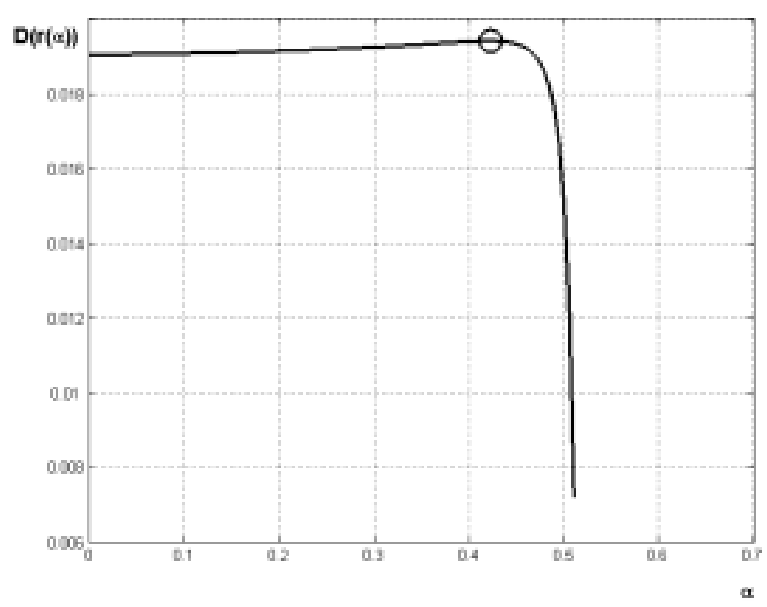

b)

Fig. 9 Fragment 5: a) image of fragment 5; b) graph of the dependence of the variance $D(r(\alpha))$ of the reconstructed image on the parameter value $\alpha$

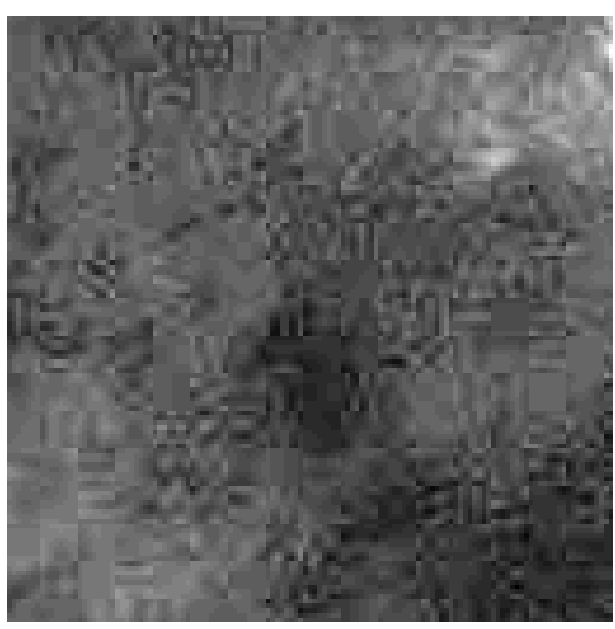

a)

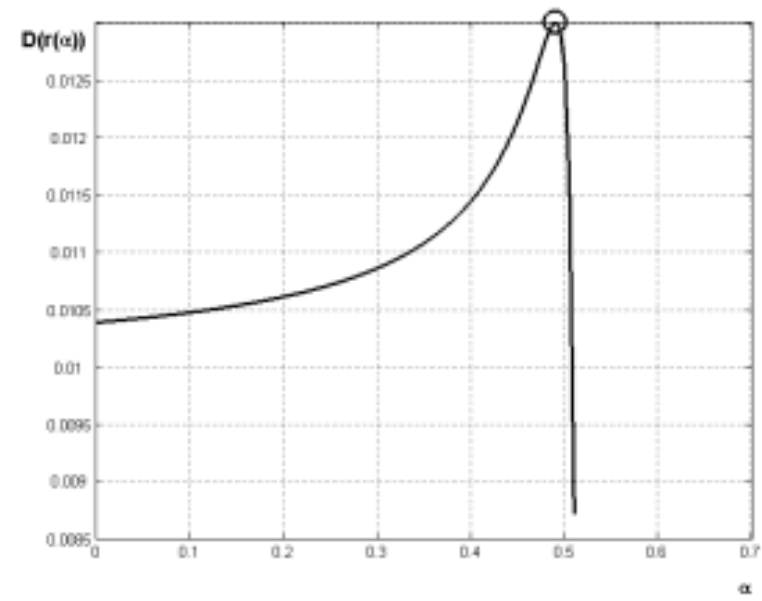

b)

Fig. 10 Fragment 6: a) image of fragment 6; b) graph of the dependence of the variance $D(r(\alpha))$ of the reconstructed image on the parameter value $\alpha$ 


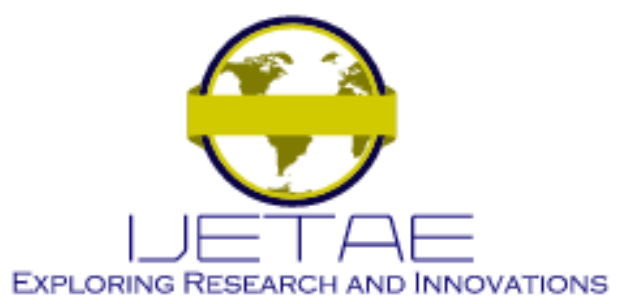

International Journal of Emerging Technology and Advanced Engineering

Website: www.ijetae.com (E-ISSN 2250-2459, Scopus Indexed, ISO 9001:2008 Certified Journal, Volume 11, Issue 11, November 2021)

In the undistorted area (no interference) $\alpha^{\prime}=0$ should always be, which is confirmed in Fig. 5-7. In this case if the found values $\alpha=\alpha^{\prime}=0$ then by formula (10) we find that $r(x, y)=\frac{1}{\gamma^{2}} g(x, y)$.

From the condition of equality of the average values of the brightness of the reconstructed image in the distorted and undistorted areas, we get that $\gamma=1$. This shows that the distortion model (8), despite its simplicity, is quite adequate. The model correctly reflects the main features of distortions caused by haze of various origins.

Let us estimate the brightness of the reconstructed image $r(x, y)$ from the registered one $g(x, y)$. The estimation will be carried out at different values of the reflection coefficient $\alpha$. The value of the multiplier $\gamma$, which takes into account the passage between the screen and the underlying surface, will be taken equal to $1(\gamma=1)$.

The calculations will be carried out according to the formula (10). Image brightness conversion curves calculated by formula (10) for various values of the coefficient $\alpha$ are shown in Fig. 11 .

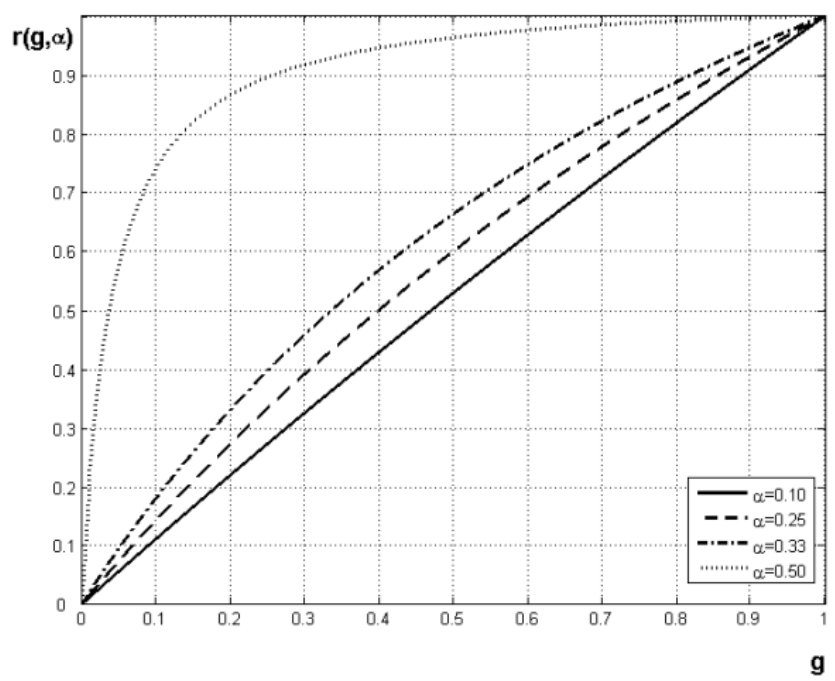

Fig. 11 The curves calculated by formula (10) for various values of the coefficient $\alpha(\gamma=1)$

From the analysis of Fig. 11 it can be seen that for small values of the coefficient $\alpha(\alpha=0,1)$, the distortions are small and $\mathrm{r}(\mathrm{g}(\mathrm{x}, \mathrm{y})) \sim \mathrm{g}(\mathrm{x}, \mathrm{y})$.

With an increase in the value of the coefficient $\alpha$, the brightness of the images differs significantly. This is due to the presence of an extended multiplicative interference.
This kind of interference is taken into account by the developed image model.

Thus, a improved imaging model in the presence of multiplicative spatially extended cloaking interference has been developed. The model take into account the effect of multiplicative masking interference.

Unlike the known ones, the model takes into account the concentration of the distorting medium in a narrow squat layer, the primary reflection of solar radiation from the upper boundary of the distorting layer and subsequent multiple re-reflections of the transmitted radiation of the visible wavelength range from the earth's surface and the upper boundary of the distorting medium layer.

A technique for finding and taking into account the reflection and re-reflection coefficients of radiation to restore distorted images is proposed.

\section{CONCLUSIONS}

Thus, the paper proposes an improved imaging model in the presence of multiplicative spatially extended cloaking interference. The model take into account the effect of multiplicative masking interference. To simplify the calculations of the image brightness in the distorted region the diagram technique is used. Unlike the known ones, the model takes into account the concentration of the distorting medium in a narrow squat layer, the primary reflection of solar radiation from the upper boundary of the distorting layer and subsequent multiple re-reflections of the transmitted radiation of the visible wavelength range from the earth's surface and the upper boundary of the distorting medium layer. A technique for finding and taking into account the reflection and re-reflection coefficients of radiation to restore distorted images is proposed. The results of experimental studies are presented. For the experiment, the image of the territory of Iraq during the 2003 "Freedom for Iraq" hostilities was selected.

Further research can be aimed at developing a method of image processing in the presence of multiplicative spatially extended cloaking interference.

\section{REFERENCES}

[1] Gonzalez, R., Woods R. Digital Image Processing. 4th Edition, Prentice Hall, Upper Saddle Rever, 2017. 1192 p.

[2] Richards, J. Remote Sensing Digital Image Analysis. An Introduction. Berlin: Springer, 2013. 159 p. doi: https://doi.org/10.1007/978-3-642-30062-2. 


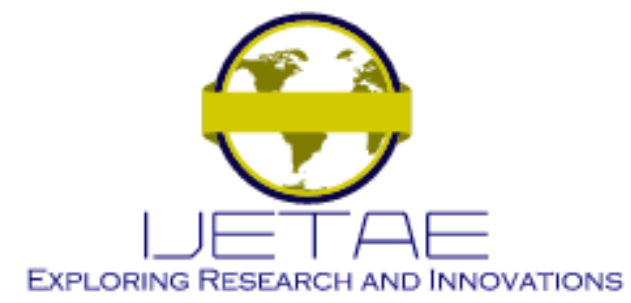

International Journal of Emerging Technology and Advanced Engineering

Website: www.ijetae.com (E-ISSN 2250-2459, Scopus Indexed, ISO 9001:2008 Certified Journal, Volume 11, Issue 11, November 2021)

[3] Ruban, I., Khudov, V., Khudov, H., Khizhnyak, I. An improved method for segmentation of a multiscale sequence of optoelectronic images. 2017 4th International Scientific-Practical Conference Problems of Infocommunications. Science and Technology (PIC S\&T). 2017. P. $137-141$. https://doi.org/10.1109/infocommst.2017.8246367.

[4] Ruban, I., Khudov, V., Makoveichuk, O., Khudov, H., Khizhnyak, I. A Swarm Method for Segmentation of Images Obtained from OnBoard Optoelectronic Surveillance Systems. 2018 International Scientific-Practical Conference Problems of Infocommunications. Science and Technology (PIC S\&T). 2018. P. 613-618. doi: https://doi.org/10.1109/infocommst.2018.8632045.

[5] Karamti, H. Tmar, M., Gargouri, F. A new vector space model for image retrieval. Procedia Computer Science, 112, 2017. P. 771-779. doi: https://doi.org/10.1016/j.procs.2017.08.202.

[6] Ballish, Bradley A., V. Krishna Kumar (November 2008). Systematic Differences in Aircraft and Radiosonde Temperatures". Bulletin of the American Meteorological Society. 89 (11): 16891708. Bibcode: 2008BAMS...89.1689B. doi:10.1175/2008BAMS2332.1. Retrieved 2011-02-16.

[7] Ruban, I., Khudov, H., Khudov, V., Khizhnyak, I., Makoveichuk, O. Segmentation of the images obtained from onboard optoelectronic surveillance systems by the evolutionary method. Eastern-European Journal of Enterprise Technologies. 2017. 5. 9 (89). 49-57. doi: https://doi.org/10.15587/1729-4061.2017.109904.

[8] Christine Aebi. C., Gröbner, J., Kazadzis, S., Vuilleumier, L., Gkikas, A., Kämpfer, N. Estimation of cloud optical thickness, single scattering albedo and effective droplet radius using a shortwave radiative closure study in Payerne. Atmospheric Measurement Techniques. 2019. doi: https://doi.org/10.5194/amt2019-347. Retrieved at: https://amt.copernicus.org/preprints/amt2019-347/amt-2019-347.pdf

[9] Xun, L.; Lu, H.; Qian, C.; Zhang, Y.; Lyu, S.; Li, X. Analysis of Aerosol Optical Depth from Sun Photometer at Shouxian, China. $\begin{array}{lll}\text { Atmosphere } & 2021, & 1226 .\end{array}$ https://doi.org/10.3390/atmos12091226.

[10] Oxford Dictionary of Biochemistry and Molecular Biology (2 ed.) Edited by Richard Cammack, Teresa Atwood, Peter Campbell, Howard Parish, Anthony Smith, Frank Vella, and John Stirling Publisher: Oxford University Press Print Publication Date: 2006 Print ISBN-13: 9780198529170. Published online: 2008 eISBN: 9780191727641

[11] Laven, Ph. Atmospheric glories: Simulations and observations, Appl. Opt. 2005. 44, 5667.

[12] Bradley, M., Gasseller, M. Measurement of Aerosols Optical Thickness of the Atmosphere using the GLOBE Handheld Sun Photometer. J. Vis. Exp. (147), 2019. e59257, doi:10.3791/59257.

[13] Bhawar RL, Fadnavis S, Kumar V, Rahul PRC, Sinha T and Lolli S (2021) Radiative Impacts of Aerosols During COVID-19 Lockdown Period Over the Indian Region. Front. Environ. Sci. 9:746090. doi: 10.3389/fenvs.2021.746090.

[14] Christensen, M. W., Jones, W. K., Stier, P. Aerosols enhance cloud lifetime and brightness along the stratus-to-cumulus transition. Proc. Natl. Acad. Sci. USA 117, 17591-17598 (2020). doi: https://doi.org/10.1073/pnas.1921231117.
[15] Khudov, H., Oleksenko, O., Lukianchuk, V., Herasymenko, V., Yaroshenko, Y., Ishchenko, O., Ikaiev, D., Golovchenko O., Volobuiev, A., Drob, Y., Solomonenko, Y., Khizhnyak, I. The Determining the Flight Routes of Unmanned Aerial Vehicles Based on Swarm Methods. International Journal of Emerging Technology and Advanced Engineering. 2021. Vol. 11. Issue 09, pp. 23-32. DOI: 10.46338/ijetae0921_03.

[16] Khudov, H., Ruban, I., Lysytsya, V., Kuzyk, P., Symkanych, O., Khudov, R The Method for Determination of Bone Marrow Cells in Photographic Images, International Journal of Emerging Trends in Engineering Research, Vol. 8. № 9, 2020, pp. 5681-5687. DOI: https://doi.org/10.30534/ijatcse/2020/131892020.

[17] Khudov, H., Symkanych, O., Kovalenko, A., Kabus, N., Lysytsya, V., Khudov, R. The Comparative Assessment of the Quality of Cytological Drugs Image Processing, International Journal of Advanced Trends in Computer Science and Engineering, Vol. 9. № 5, 2020, pp. 8645-8653. DOI: https://doi.org/10.30534/ijatcse/2020/250952020.

[18] Ruban I., Khudov H., Makoveychuk O., Khizhnyak I., Khudov V., and Lishchenko V. The model and the method for forming a mosaic sustainable marker of augmented reality. Advanced Trends in Radioelectronics, Telecommunications and Engineering (TCSET): Thesis of 2020 IEEE 15th International Conference (Slavske, February 25-29, 2020). Slavske: IEEE, 2020. P. 402-406. DOI: https://doi.org/10.1109/TCSET49122.2020.235463.

[19] Ruban, I., Makoveychuk,O., Khudov, V., Khizhnyak, I., Khudov, H., Baranik, O. The Mosaic Sustainable Marker Detection Method for Augmented Reality Systems, in Intern. Scient.-Pract. Conf. Problems of Infocommunications. Science and Technology (PIC S\&T), 2020, pp. 311-317. https://doi.org/10.1109/PICST51311.2020.9467962.

[20] Khudov, H., Makoveychuk, O., Khizhnyak, I., Yuzova, I., Irkha, A., Khudov, V. The mosaic sustainable marker model for augmented reality systems, Internetional Journal of Advanced Trends in Computer Science and Engineering, 2020. № 9(1), 637-642. DOI: https://doi.org/10.30534/ijatcse/2020/89912020.

[21] Ruban, I., Khudov, H. Advances in Spatio-Temporal Segmentation of Visual Data, Chapter 2. Swarm Method of Image Segmentation. Series Studies in Computational Intelligence (SCI), Vol. 876. Publisher Springer, Cham, $2020 . \quad-\quad$ P. 53-99. DOI https://doi.org/10.1007/978-3-030-35480-0.

[22] Ruban, I., Makoveichuk, O., Khudov, V., Khizhnyak, I., Khudov, H., Yuzova, I., Drob, Y. The Method for Selecting the Urban Infrastructure Objects Contours, in Intern. Scient.-Pract. Conf. Problems of Infocommunications. Science and Technology

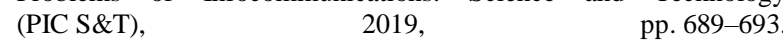
DOI: https://doi.org/10.1109/infocommst.2018.8632045.

[23] Khudov, H., Khizhnyak, I., Koval, V., Maliuha, V., Zvonko, A., Yunda, V., Nagachevskyi, V., Berezanskyi, V. The Efficiency Estimation Method of Joint Search and Detection of Objects for Surveillance Technical Systems, International Journal of Emerging Trends in Engineering Research, Vol. 8. № 3, 2020, pp. 813-819. DOI: https://doi.org/10.30534/ijeter/2020/34832020.

[24] The Cambridge dictionary of statistics / B.S. Everitt, A. Skrondal. Everitt, Brian. Cambridge, UK ; New York : Cambridge University Press; 2010.

[25] Retrieved at: http://www.spaceimaging.com/ gallery/default.htm. 